http://doi.org/10.15359/ree.14-1.15

\title{
Espacios vitales de aprendizaje: su ecología en el sétimo año de la educación pública costarricense
}

\author{
Magdalena Alfaro Rodríguez, \\ Centro de Investigación y Docencia en Educación (CIDE), Universidad Nacional \\ Heredia, Costa Rica \\ Alejandra Gamboa Jiménez ${ }^{2}$ \\ Centro de Investigación y Docencia en Educación (CIDE), Universidad Nacional \\ Heredia, Costa Rica \\ Susana Jiménez Sánchez. \\ Centro de Investigación y Docencia en Educación (CIDE), Universidad Nacional \\ Heredia, Costa Rica \\ Jorge R. Martín Pérez ${ }^{4}$ \\ Centro de Investigación y Docencia en Educación (CIDE), Universidad Nacional \\ Heredia, Costa Rica \\ Andrea Ramírez González ${ }^{5}$ \\ Centro de Investigación y Docencia en Educación (CIDE), Universidad Nacional \\ Heredia, Costa Rica \\ Marie-Claire Vargas Dengo 6 \\ Centro de Investigación y Docencia en Educación (CIDE), Universidad Nacional \\ Heredia, Costa Rica
}

\footnotetext{
${ }^{1}$ Magíster en Educación con énfasis en Docencia Universitaria de la Universidad Nacional, Costa Rica. Licenciada en Música con énfasis en Educación Musical. Actualmente, es académica de la División de Educología del Centro de Investigación y Docencia en Educación (CIDE) de la Universidad Nacional, Costa Rica, y es integrante del cuarto equipo del Proyecto Perfiles, dinámicas y desafíos de la educación costarricense. Correo electrónico: magdar613@hotmail.com
} ${ }^{2}$ Máster en Psicopedagogía de la Universidad de La Salle, con estudios en Ciencias de la Educación con énfasis en Orientación de la Universidad
Nacional, Costa Rica, actualmente es la subdirectora de la División de Educación para el Trabajo del Centro de Investigación y Docencia en
Educación (CIDE) de la Universidad Nacional, Costa Rica y fue integrante del tercer equipo del proyecto Perfiles, dinámicas y desafíos de la
educación costarricense. Correo electrónico: 1gamboa@una.ac.cr

${ }^{3}$ Máster en Tecnología Educativa de la Universidad Estatal a Distancia, Costa Rica. Licenciada en Enseñanza de la Química de la Universidad
de Costa Rica y en Ciencias de la Educación con énfasis en Docencia de la Universidad Estatal a Distancia, Costa Rica. Con estudios en edu-
cación primaria. Actualmente, es académica de la División de Educología del Centro de Investigación y Docencia en Educación (CIDE) de la
Universidad Nacional, Costa Rica y coordinó el tercer equipo del proyecto Perfiles, dinámicas y desafíos de la educación costarricense. Correo
electrónico: sujisa@gmail.com

${ }^{4}$ Magíster en Educación con énfasis en Docencia Universitaria de la Universidad Nacional, Costa Ria, Licenciado en Economía y Licenciado en Cibernética Matemática de la Universidad de la Habana, Cuba. Actualmente, es académico de la División de Educación para el Trabajo del Centro de Investigación y Docencia en Educación (CIDE) de la Universidad Nacional, Costa Rica y coordina el cuarto equipo del proyecto Perfiles, dinámicas y desafíos de la educación costarricense. Correo electrónico: jorgeramonmartin@yahoo.es

${ }^{5}$ Licenciada en Enseñanza de la Matemática de la Universidad de Costa Rica y con estudios en Educación Primaria. Actualmente, es académica de la División de Educación Rural del Centro de Investigación y Docencia en Educación (CIDE) de la Universidad Nacional, Costa Rica y fue integrante del tercer equipo del proyecto Perfiles, dinámicas y desafíos de la educación costarricense.

Correo electrónico: aramgo@costarricense.cr

${ }^{6}$ Máster en Currículum e Instrucción de The George Washington University, USA. Actualmente, es académica de la División de Educación Básica del Centro de Investigación y Docencia en Educación (CIDE) de la Universidad Nacional, Costa Rica y es integrante del cuarto equipo del Proyecto perfiles, dinámicas y desafíos de la educación costarricense. Correo electrónico: educare@una.ac.cr

La Revista Electrónic@ Educare está indizada en el Catálogo LATINDEX.

Artículo protegido por la licencia Creative Commons. 
Resumen. Este artículo es el resultado de una investigación del CIDE-UNA para construir el perfil de docentes deseables y posibles para el $7^{\circ}$ año de la educación pública costarricense; en él se evidencia la trascendencia de la ecología de los espacios de aprendizaje, entendida como el ambiente, los actores involucrados y sus interrelaciones complejas. Se describen estos espacios, y su incidencia en la permanencia y la promoción del estudiantado, en el momento singular en que el sistema educativo les impone los primeros y bruscos cambios institucionales coincidentes con transformaciones físicas y en sus relaciones familiares y sociales.

Palabras clave. Ecología espacios aprendizaje, espacios vitales de aprendizaje, ambiente aula, clima de aula, sétimo año, educación secundaria, perfil docente.

\begin{abstract}
The present article is the result of an investigation between CIDE-UNA to build a desirable profile for teachers that might teach seventh grade in Costa Rican public education schools. This article evidences transparency in the ecology of learning spaces, known as the environment, the actors involved and their complex interrelations. There is also a description of these spaces and their incidence in the permanence and promotion of the students population, in the singular moment in which the educational system imposes them the first and rough institutional changes coinciding with physical transformations and social and familiar relationships.
\end{abstract}

Key words. Learning ecology space, environment, seventh grade, middle school, teacher profile.

\title{
Perspectiva teórica
}

El objetivo principal de este artículo es fundamentar la importancia que tienen los espacios vitales del aprendizaje. El estudio se realiza como actividad de la III etapa de investigación del Proyecto Perfiles, Dinámicas y Desafíos de la Educación Costarricense, adscrito al Decanato del Centro de Investigación y Docencia en Educación (CIDE) de la Universidad Nacional de Costa Rica (UNA), cuyo tema principal fue el perfil de desempeño docente del sétimo año, y en la que se destaca la importancia del espacio vital donde la comunidad educativa (docente y estudiantil) desarrolla los procesos de enseñanza y de aprendizaje.

Los espacios vitales de aprendizaje refieren a las dinámicas que se conjugan en una práctica pedagógica dada, involucra diversos elementos, tales como: los recursos humanos, la disposición de los recursos materiales, el manejo del tiempo y la disposición física del grupo y del mobiliario, todo lo que genera un ambiente singular de convivencia.

En la concepción integrada de los mencionados elementos, es importante conocer tanto las características individuales del estudiantado como las interrelaciones que prevalecen, para lo que se requiere conocer entornos familiares, socioeconómicos, culturales y todos los que se consideren influyentes en las actitudes que se asumen en el aula.

Según Morin (1999), en la ecología de la acción intervienen las inter-retro-acciones, tanto de la población estudiantil como de la población docente, por lo que una acción puede tener resultados inesperados para quien la realiza, a causa de que confluyen personas únicas que interpretan los aprendizajes en forma distinta. 
En los espacios vitales de aprendizaje confluyen las acciones docentes, en las que los conocimientos, la presencia y el comportamiento profesional y ético, con honestidad, afectividad y respeto, contribuyen al desarrollo integral de cada estudiante: es la insustituible fuerza del ejemplo.

Las funciones que desempeña el profesorado propician valores que permiten a sus aprendices, convivir en espacios vitales diferentes del ámbito escolar, trascienden el marco cognitivo disciplinar y resultan en habilidades adaptativas y readaptativas, para vivir en un mundo aceleradamente cambiante, diverso y globalizado.

El fundamento teórico fundamental, que respalda el tema de los espacios vitales del aprendizaje, se constituye en el modelo sistémico-ecológico de las ciencias humanas y sociales, uno de los principales autores del enfoque ecológico fue Bronfenbrenner (1987), quien en los años sesentas concibió una teoría ecológica basada, fundamentalmente, en estudios en el área de la psicología evolutiva, lo que contribuyó a las investigaciones acerca de los contextos educativos, desde el escenario natural de los aprendizajes, con un enfoque sistémico del contexto.

El estudio de la realidad del contexto, en la que interactúan grupos estudiantiles de sétimo año, involucra infinitos detalles microambientales. Tanto el estudiantado como el cuerpo docente se encuentran en constante aprendizaje, y el espacio de aprendizaje es lugar vital de construcción y reconstrucción del conocimiento, por medio de multiplicidad de intercambios, los que, a su vez, promueven los valores, las actitudes y el desarrollo de habilidades para la vida.

El espacio vital de desarrollo del aprendizaje está sujeto a continuos cambios que involucran a estudiantes y docentes, desde la percepción y la adaptación ambiental, hasta las múltiples y sistemáticas interrelaciones, las que producen una diversidad compleja con implicaciones positivas o negativas para todos los actores.

La teoría de Bronfenbrenner (1987) aporta conocimiento en cuanto a que el estudiantado y el cuerpo docente interactúan en un entorno inmediato cambiante, en progresiva adaptación a éste, y que se puede reconocer como un espacio ecológico.

En el espacio educativo, quien ejerce la docencia se va implicando e influyendo, propiciando sistemáticos cambios y adaptaciones que resultan en la diversidad de interrelaciones entre los procesos de enseñanza y de aprendizaje, y el surgimiento de múltiples ajustes y desajustes dentro de la dinámica escolar.

La visión ecológica de los espacios vitales de aprendizaje tiene una íntima relación teórica y sistémica de entendimiento de los fenómenos que surgen en ellos.

El todo, entendido como la población estudiantil, el docente o la docente, el currículo, el aprendizaje, la enseñanza, los factores de infraestructura y climáticos, el mobiliario, la distribución física, la didáctica, las relaciones interpersonales y la diversidad de cuantos elementos confluyen en este espacio, están interrelacionados. Lo que ocurra con algún elemento afecta al resto; por ejemplo, cuando se producen deserciones o incorporaciones de estudiantes o cambios de docentes e, incluso, nuevas ornamentaciones, mobiliarios o simples reordenamientos físicos se afectan los otros elementos.

Desde el punto de vista de un modelo ecológico en los espacios vitales de aprendizaje, las interacciones y transacciones que mantienen la población estudiantil y el cuerpo docente deben analizarse considerando al resto de los elementos (físicos, de ordenamiento, entre otras).

La población estudiantil se ve influida por un espacio ecológico más extenso al de su grupo educativo; al respecto, Bronfenbrenner (1987) postula cuatro niveles o sistemas funcionales que implican a la persona (véase la Figura 1). 


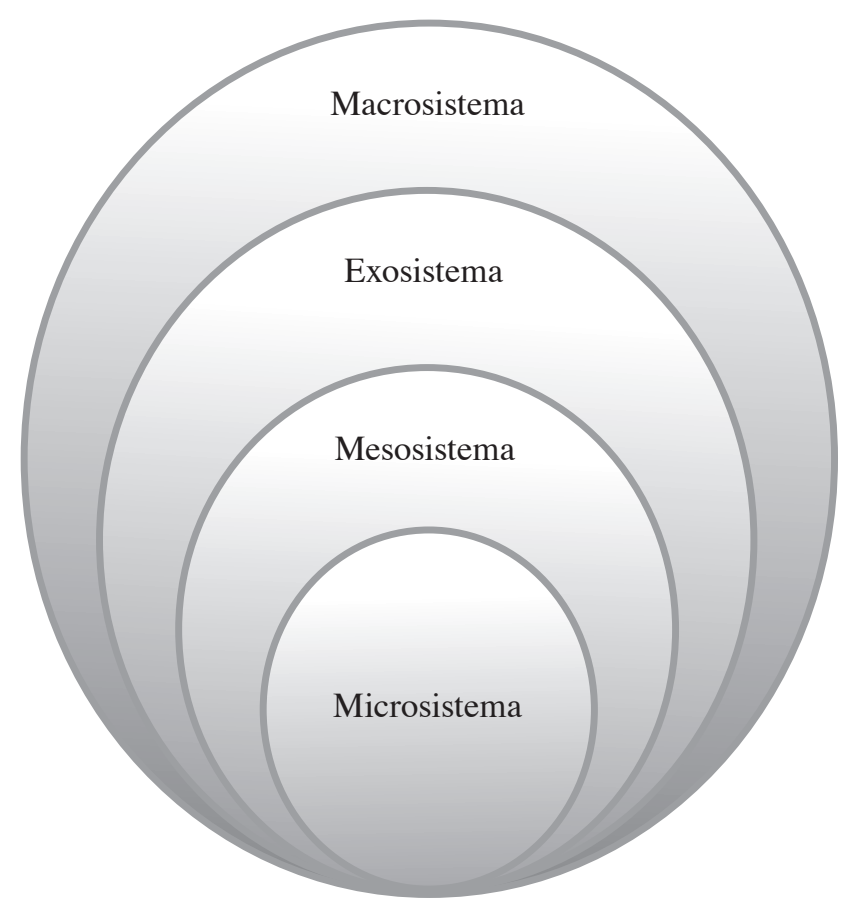

Figura 1. Sistemas desde la perspectiva ecológica (Bronfenbrenner)

Nota. Elaboración propia, a partir de la ejemplificación descrita por Bisquerra, R. (1998), como analogía de los sistemas o estructuras concéntricas del contexto (que propone Bronfenbrenner, U.) al estilo matrioskas, que son las típicas muñecas rusas que se encajan una dentro de otra.

Microsistema. Corresponde al patrón de actividades, roles y relaciones interpersonales que la persona en desarrollo experimenta en el entorno determinado en el que participa.

Mesosistema. Comprende las interrelaciones de dos o más entornos (microsistemas) en los que la persona en desarrollo participa (por ejemplo, para las personas adolescentes: las relaciones entre el hogar, el colegio y su grupo de iguales; para un adulto: entre la familia, el trabajo y la vida social).

Exosistema. Se refiere a los propios entornos (uno o más) en los que la persona en desarrollo no está incluida directamente, pero en los que se producen hechos que afectan a lo que ocurre en los entornos en los que la persona sí está incluida (para el adolescente, podría ser el lugar de trabajo de sus padres, la universidad del hermano mayor, el círculo de amigos de sus padres, entre otros).

Macrosistema. Se refiere a los marcos culturales o ideológicos que influyen, transversalmente, a los sistemas de menor orden (micro, meso y exosistema), y que les confiere a éstos una cierta uniformidad, en forma y contenido y, a la vez, una cierta diferencia, con respecto a otros entornos influidos por otros marcos culturales o ideológicos diferentes.

Por lo anterior, el colectivo discente ve reflejado en el espacio educativo los otros ambientes, los cuales desarrolla diariamente $y$, consecuentemente, es importante que su docente los tenga en cuenta, ya que conforma parte de las interacciones que se dan en la vida del grupo.

\section{Uso de los espacios físicos y el tiempo en el aula}

El espacio físico conocido como "aula", proporciona elementos que pueden utilizarse para armonizar el espacio vital de aprendizaje, y motivar al estudiantado con temas, contenidos u 
objetivos específicos, estrategias de aprendizajes intencionales que fomenten el conocimiento. Por otro lado, espacialmente, la ubicación de los elementos que, por lo general, se encuentran en el salón de clases, deben propiciar la inclusión de la población discente y evitar la exclusión de alguna persona; asimismo, debe promover la participación y el libre desplazamiento, basado en el respeto y las reglas del grupo.

El espacio físico debe tener un propósito principal y éste lo define, intencionalmente, la persona docente de acuerdo con los objetivos que se plantea y según sus habilidades, no hay una distribución de asientos que sea ideal para todas las clases, todas las situaciones de aprendizaje y todos los estudiantes.

Por ejemplo, la distribución en filas en la que un estudiante se sienta detrás del otro mirando hacia la pizarra, (véase figura 2) es funcional cuando la persona docente quiere inhibir la interacción entre estudiantes; puede resultar adecuada para trabajos individuales diversos, en los cuales no se requiere ningún tipo de interacción de unos estudiantes con otros, para realizar una demostración o un examen individualizado, o alguna conferencia; sin embargo, no se considera la mejor cuando el proyecto es de tipo cooperativo.

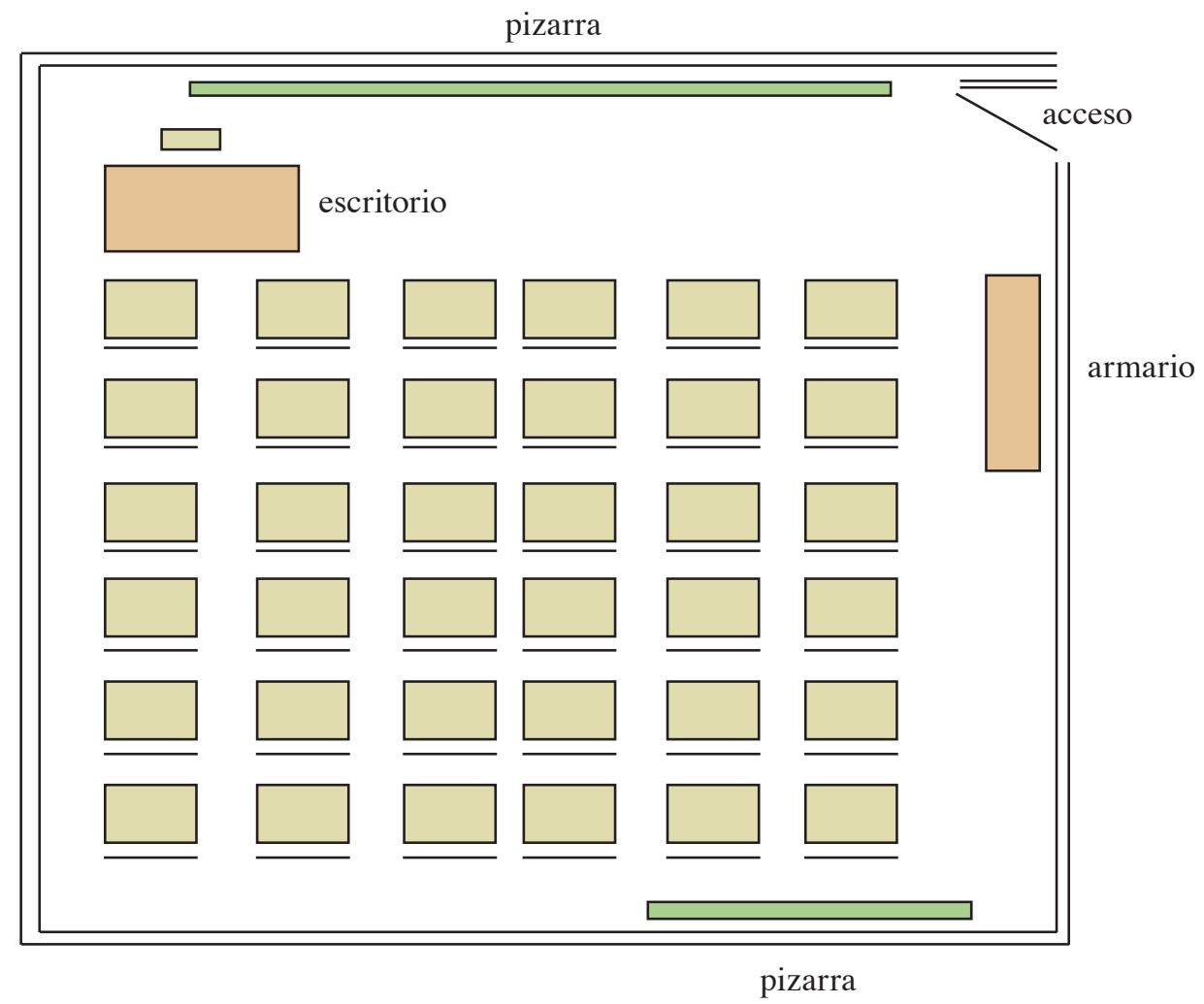

Figura 2. Distribución lineal de pupitres.

Nota. García, N., Rojas, M., Campos, N., Brenes, M., Campos, E. (1993). Conocimiento, participación y cambio: capacitación a docentes a partir de la investigación en el aula. San José, Costa Rica: EUCR.

La persona docente puede elegir formaciones semipermanentes, que sirvan para varias actividades en el aula, y realizar formaciones especiales que aporten variedad al espacio vital de aprendizaje y cumplir con las necesidades de una tarea determinada. 
La distribución de los asientos, en el aula, puede favorecer tanto metas pedagógicas como determinados comportamientos del alumnado. La distribución de los asientos tiene que ser compatible con el tipo de actividad y con las necesidades de los estudiantes" (Candegabe, 2001).

Para García et al. (1993), la construcción de un concepto de uso eficiente del espacio vital de aprendizaje, toma en cuenta el espacio como un elemento participante en la comunicación que se establece dentro del aula y considera que debe ser flexible, mediante la adecuada distribución de pupitres, en relación con los objetivos, las actividades de aprendizaje y la interacción que se pretenden establecer.

El modelo ecológico de los espacios vitales de aprendizaje sugiere la distribución de pupitres en círculo (véase la Figura 3). Esta forma contribuye en la interacción de las personas, la cooperación se torna más efectiva y puede promoverse el trabajo grupal y de equipo; promueve, también, el intercambio cognitivo y de experiencias, pues favorece los lazos afectivos y el sentido de pertenencia al grupo; su fortaleza radica en el contacto visual directo que provoca la ubicación de las personas que intenta desinhibir y humanizar las relaciones.

\section{pizarra}

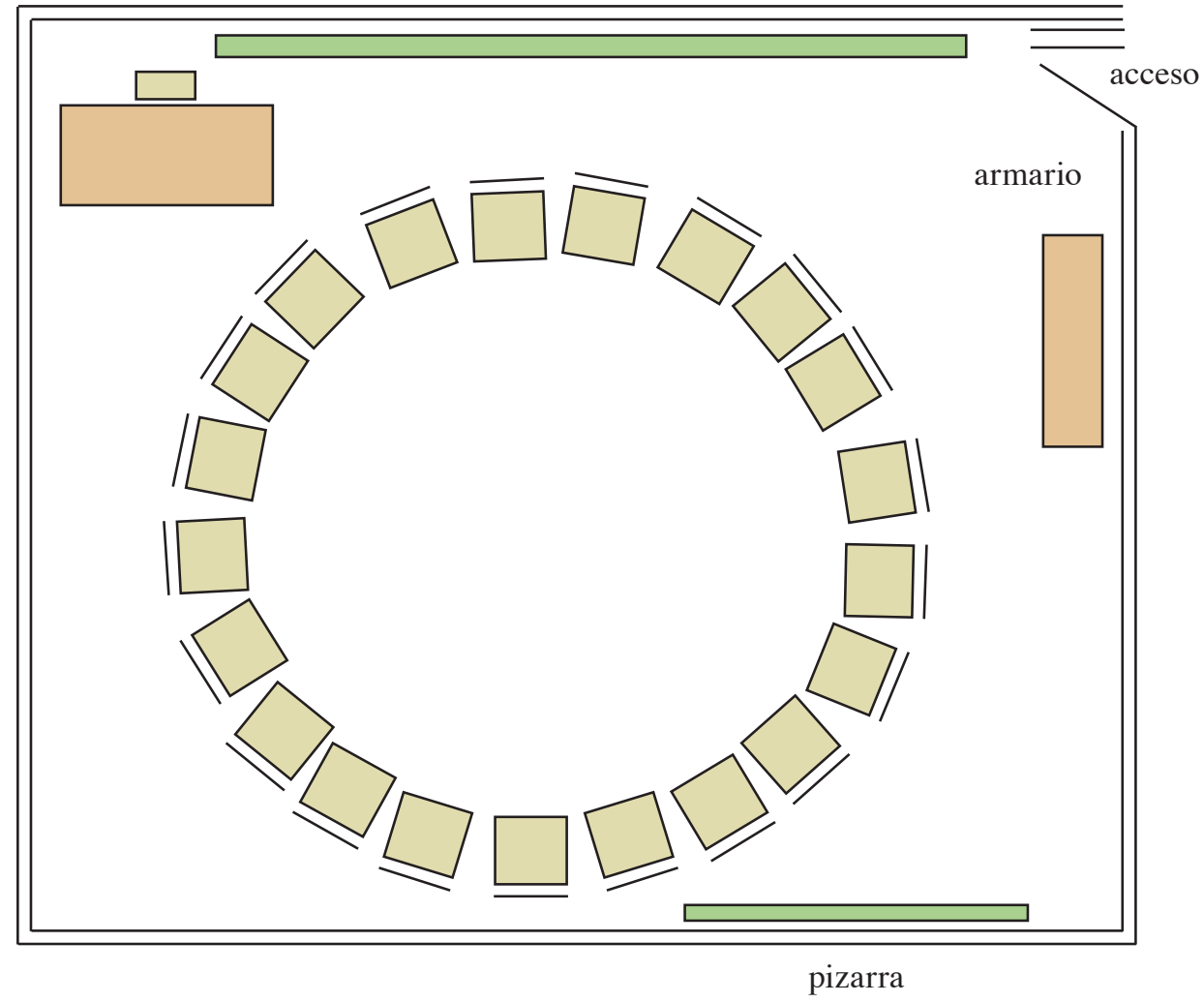

Figura 3. Distribución circular de pupitres.

Nota. García, N., Rojas, M., Campos, N., Brenes, M., Campos, E. (1993). Conocimiento, participación y cambio: capacitación a docentes a partir de la investigación en el aula. San José, Costa Rica: EUCR.

Además, desde este modelo se sugiere la distribución de pupitres en semicírculo (véase la Figura 4), la cual puede utilizarse con dos modalidades, dependiendo del número de participantes y del objetivo y del tipo de actividades que se propone. Los pupitres pueden organizarse en un solo semicírculo, o colocarse de manera que formen varios de ellos. 


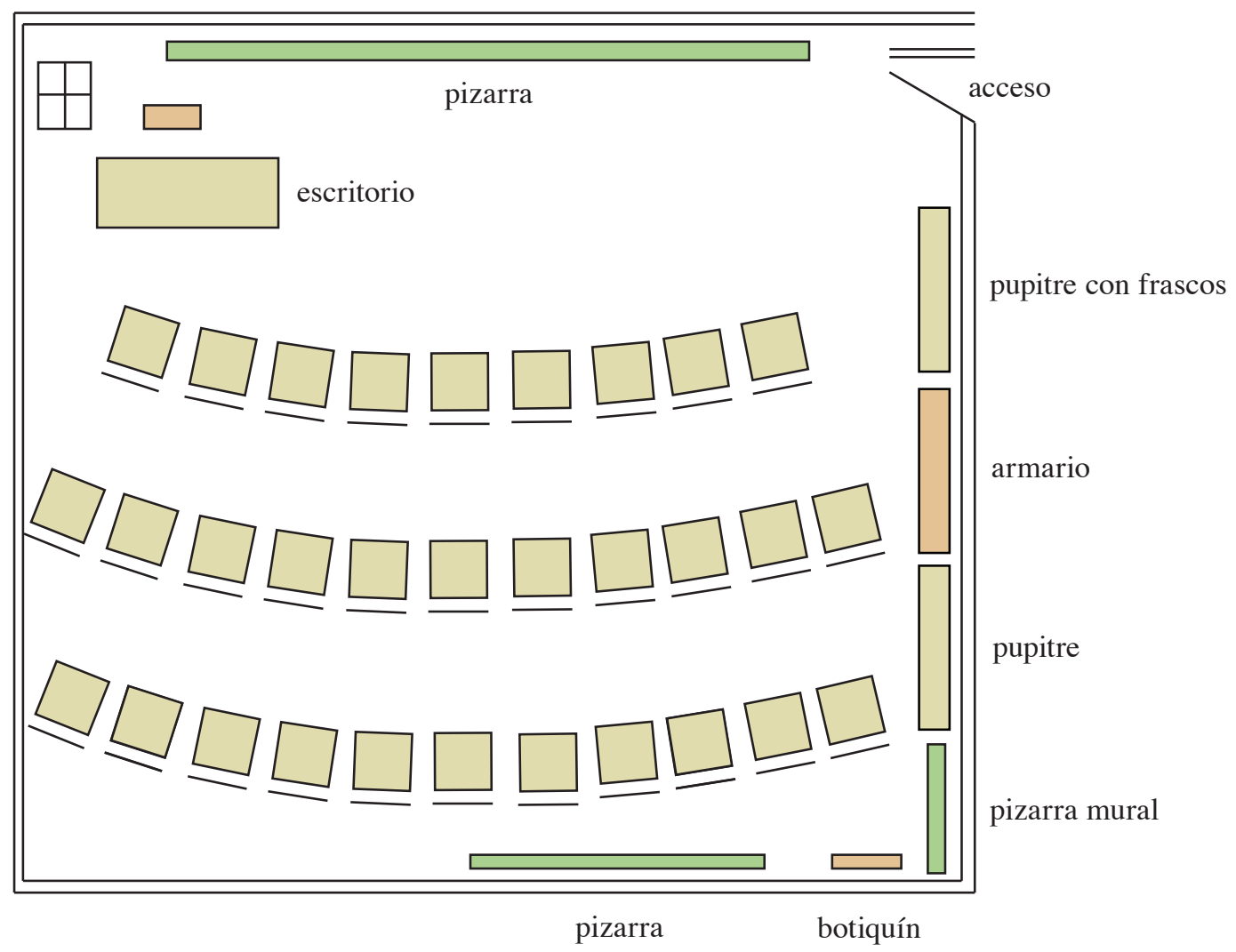

Figura 4. Distribución semicircular de pupitres.

Nota. García, N., Rojas, M., Campos, N., Brenes, M., Campos, E. (1993). Conocimiento, participación y cambio: capacitación a docentes a partir de la investigación en el aula. San José, Costa Rica: EUCR.

Este tipo de distribución es favorable en actividades de charlas o conferencias, en las que se exhibe material didáctico o se proyectan películas, diapositivas, vídeos, pues permite a la población estudiantil una mejor visibilidad del recurso didáctico que se utiliza y una interacción más cercana de la población estudiantil a la exposición de un tema específico.

En relación con la distribución de pupitres en grupos (véase la Figura 5), se utiliza, principalmente, cuando se desea propiciar un trabajo de tipo cooperativo entre la población estudiantil, pues se ubican mirándose cara a cara y se promueven interacciones en un círculo de estudiantes más pequeño y cercano.

Dentro de los beneficios de esta distribución para la promoción de los aprendizajes se encuentra:

1. Facilita el acceso al material de interés en las diferentes actividades de aprendizaje.

2. Permite tanto la participación del estudiantado en las actividades propuestas, como la realimentación del aprendizaje del alumnado, por parte del maestro o la docente.

3. Permite el desplazamiento fácil y responsable de los y las estudiantes en el aula.

4. Establece zonas particulares para trabajos específicos dentro del espacio de aprendizaje.

5. Estimula nuevas formas de aprender y reaprender gracias a que provee espacios concretos para la exposición del trabajo.

6. Incorpora espacios de aprendizaje extra-aula al quehacer o trabajos de clase. 


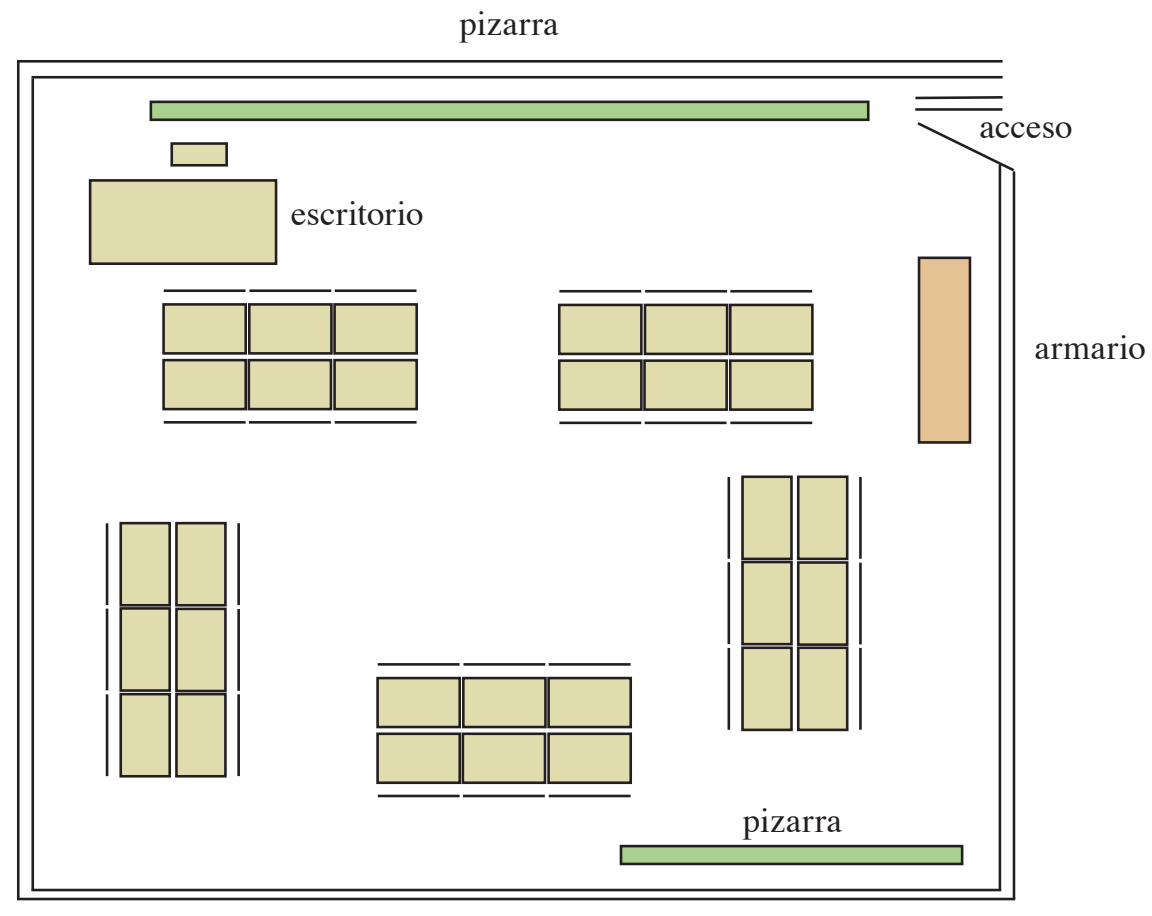

Figura 5. Distribución de pupitres en grupos.

Nota. García, N., Rojas, M., Campos, N., Brenes, M., Campos, E. (1993). Conocimiento, participación y cambio: capacitación a docentes a partir de la investigación en el aula. San José, Costa Rica: EUCR.

En cuanto al uso eficiente del tiempo, se consideran algunos aspectos que pueden favorecer los procesos de enseñanza y de aprendizaje:

1. La planificación del tiempo en el aula, por lo general, es controlada por la persona que realiza la mediación en el proceso educativo, de acuerdo con los objetivos que se plantea: sin embargo, se sugiere dar a conocer lo planificado para la clase o sesión de encuentro y ponerlo de mutuo acuerdo.

2. En ocasiones, las lecciones son planificadas en momentos del día cuando hace más calor o más frío, o después de la comida u otros factores, por tanto, se recomienda tener en cuenta estos elementos, porque influyen en la concentración y, por ende, en el uso óptimo del tiempo real para los aprendizajes.

3. La planificación del tiempo debe considerar la diversidad de ritmos de la población estudiantil y los posibles trabajos alternativos que se puedan realizar, para lograr los mismos objetivos en el tiempo con el que se cuenta.

Existe un elemento dentro de la planificación conocido como el "principio Eisenhower, este famoso general fue quien dirigió el desembarco de las tropas de los Aliados en Normandía y más tarde fue presidente de Estados Unidos. Para organizar sus numerosas tareas, acostumbraba a repartirlas en cuatro categorías: importante y urgente, importante y no urgente, no importante y urgente, no importante y no urgente" (Boeglin, 2008, p. 43), se considera que esta experiencia que se dio en otro contexto podría contribuir para el buen uso del tiempo que se distribuye en el espacio vital de aprendizaje. 
Todo lo relacionado con la distribución del espacio y del tiempo es vinculante, tanto a las características del estudiantado como al perfil docente de desempeño.

\section{Consideraciones metodológicas}

La investigación que dio origen a este artículo utilizó un enfoque metodológico cualitativo, con apoyo de informaciones cuantitativas existentes, por medio de estrategias descriptivo-interpretativas.

La recopilación de la información inexistente se realizó utilizando observaciones de aula, entrevista a docentes y estudiantes de sétimo año y talleres con estudiantes y profesores de este nivel, así como entrevistas complementarias a orientadores y directores en una muestra intencionada de nueve centros secundarios públicos de zonas urbanas y rurales de las provincias de San José, Guanacaste y Cartago.

\section{Resultados y discusión}

Para el análisis de los resultados de la dimensión correspondiente a la ecología de los espacios vitales de aprendizaje, los principales aspectos identificados fueron los actitudinales, tamaños de grupos, condiciones físicas y ambientales, interrupciones y elementos distractores, afectividad, respeto y manejo de límites, cooperación y motivación.

Existe una estrecha relación entre la ecología en los espacios vitales de aprendizaje, la dinámica del grupo, las actitudes propias de estudiantado de sétimo año y el desempeño docente.

Es casi generalizada la distracción coloquial entre estudiantes en pleno desarrollo lectivo, sin orden ni concierto y ajena a la temática, casi ignorando la presencia y las acciones de sus docentes. Igualmente, tirarse cosas, deambular por el "aula", entrar y salir con frecuencia de ésta por necesidades sanitarias, entre otras.

Hay relación directa entre la motivación y la funcionalidad o la comprensión de las actividades propuestas y la atención y participación del estudiantado; todo lo que es muy evidente, se presentó, también, en otro extremo no deseable, un ambiente poco afectado por elementos distractores, el que es impuesto por la persona docente, pero, en este caso, se denota pasividad poco participativa como parte del silencioso y, aparentemente, respetuoso ambiente imperante.

En cuanto al "aprovechamiento del tiempo", se apreció también bastante generalizada actitud de desaliento estudiantil, con frecuencia de impuntualidades y deseos manifiestos de terminar, anticipadamente, las clases o ansias de cualquier factor de suspensión de ellas.

Los trabajos en grupo son una posibilidad de socializar de manera entusiasta, lo que es una necesidad en el grupo de edad en estudio, y es, a su vez, una forma de intercambiar ideas, esto puede lograrse con un enfoque de disciplina integradora, participativa y no silenciosa y pasiva.

La pasividad inhibe el intercambio de ideas y la construcción cooperativa de conocimiento, con bajo desarrollo de la creatividad. La población estudiantil requiere una mediación pedagógica atractiva para su edad.

En las disciplinas de cómputo o informática, la propia atracción de los medios técnicos propician ambientes más motivadores de aprendizaje y, en general, se observó que la interacción docenteestudiantes y entre pares es más favorable. Generalmente, el "aula" está climatizada y se propician logros individuales y colectivos evidenciados en cada sesión, hay más interés el cual se manifiesta en constantes interrogantes temáticas del estudiantado a sus docentes. 
Es evidente el efecto positivo de las tecnologías más avanzadas como elementos de la ecología del espacio educativo, lo que se aprecia cuando se utilizan vídeos o medios audiovisuales para las exposiciones.

Uno de los desafíos en el aula para el cuerpo docente radica en interesar a sus estudiantes en la temática en estudio, porque, de lo contrario, se manifiestan desafiantes o con dedicación temporal al cumplimiento de las metas del momento, con el único objetivo de obtener las evaluaciones que se proponen.

Otras manifestaciones indeseadas, pero frecuentes en las realidades observadas en los espacios vitales de aprendizaje, son las constantes llamadas de atención de docentes a estudiantes, burlas o irrespetos de los segundos a los primeros y a sus pares, vocabulario soez, entre otras.

Otros elementos distractores en la dinámica frecuente de los espacios vitales de aprendizaje se relacionan con la ingestión de alimentos o bebidas, el maquillado propio o entre pares, el intercambio de mensajes ajenos a la clase en papeles, mediante celulares, el uso de tecnologías de disfrute individual de música o las simples conversaciones.

En relación con aspectos evaluativos, en lugar de propiciar lo formativo, se utiliza, con mucha frecuencia, punitivamente, mediante la aplicación de instrumentos de medición sorpresivos, la mayor asignación de "tareas extraclase" y la emisión de boletas, todo ello como forma de "llamado al orden”, pero que provocan rechazo a las pruebas, exámenes y tareas, con temor o burla por las calificaciones y la pérdida de motivación de este elemento tan importante para la construcción del aprendizaje como es la evaluación, concebida con integralidad y contextualización.

En la mayoría de los casos, el estudiantado que se interesa por obtener elevados promedios, lo hace más por la repercusión social y familiar que por real motivación de aprendizaje; esto último se evidencia en muy pocos casos.

Algunos otros comportamientos del estudiantado o interrupciones, que con frecuencia afectan la ecología de los espacios vitales de aprendizaje, son:

- Respuesta limitada a lo que pregunta el profesor

- Destrucción o maltrato del mobiliario

- Saludos entre pares no practicados entre docente-estudiantes

- Solicitud de cooperación ante dudas, preferentemente, entre pares más que a docentes

- Uso de sobrenombres o apodos

- Silencio como respuesta a preguntas grupales de docentes

- Desacato a instrucciones de docentes

- Salida de estudiantes a ensayos, para actividades extracurriculares

- Convocatoria por altavoces o a viva voz interrumpiendo lecciones

- Reuniones o contactos de organizaciones estudiantiles o gremiales

- Ocupación de estudiantes o docentes en rifas u otras actividades similares

- Comunicaciones orales o escritas para los docentes en plena lección 
Muchas direcciones de centros educativos, dirigentes estudiantiles y gremiales, lejos de ser garantes del buen desarrollo del proceso educativo no promueven espacios vitales de aprendizaje óptimos para el desarrollo integral de la comunidad estudiantil.

Para minimizar los efectos negativos señalados, algunos docentes desarrollan su lección con aplicación intensiva de actividades y, hasta, exigen tareas extraescolares que ocupen a su estudiantado vinculado disciplinarmente, aunque esto no garantiza la motivación y el consecuente aprendizaje significativo, y puede, incluso, provocar mayores rechazos, por lo que si bien el aprovechamiento del tiempo es fundamental, éste debe concebirse con suficiente motivación y alternancia de técnicas, para evitar la monotonía y el aburrimiento.

Existen grupos en los que se da mayor participación por parte del estudiantado, sobre todo, cuando la persona docente logra un acercamiento personal y fomenta relaciones flexibles, y no se castiga el error, pero sí se reconocen los logros y se estimula el esfuerzo.

La receptividad del docente es muy positiva, su disposición a aclarar, repetir, no reprender, sino razonar, su atención a cada demanda o necesidad de cualquier estudiante, el estímulo y reconocimiento de la cooperación, en aras de lograr los objetivos compartidos, sobre la base de la confianza y el respeto mutuos, pero todo ello partiendo de las acciones personales ejemplarizantes que ofrece el cuerpo docente.

El ambiente que se da en las relaciones debe generarse, conscientemente, a partir del liderazgo que sea capaz de quien ejerce la docencia. Si es permisivo tienden a perderse los límites, se afecta el respeto mutuo y, como consecuencia, se pierde el "encanto necesario para propiciar aprendizajes". Si, por el contrario, es autoritario, probablemente, se genere pasividad y apatía, si no, rechazo directo. Un ejercicio docente democrático, participativo y respetuoso, casi siempre, fructifica en motivación, cooperación y compromiso del estudiantado con los objetivos de aprendizaje.

El espacio vital de aprendizaje, también, es determinado por todos los aspectos externos que puedan ser percibidos por cualesquiera de los sentidos del estudiantado, por lo que, cuando afectan el desempeño docente deseado, debe recurrirse a las autoridades o mecanismos que puedan eliminar o minimizar los efectos negativos, además de procurar potenciar elementos internos controlables por la o el docente, que puedan también neutralizarlos o revertirlos. En este caso, además de cuestiones tangibles (sonidos, olores, temperaturas, etc), se incluyen otros de más difícil detección, tales como temores, tentaciones, entre otros.

En algunas instituciones, se encuentran zonas verdes que refrescan las aulas y permiten un clima agradable y fresco, aunque en otras el exceso de calor tiene implicaciones en el estado anímico tanto de la comunidad docente como de la estudiantil.

La limpieza, la pintura, la ambientación alusiva a la temática, la ornamentación agradable, no recargada y acorde con la estética imperante en el grupo etario del estudiantado, son una importante contribución a la ecología del espacio educativo.

La distribución lineal de los pupitres es la organización física imperante, como una posibilidad de mantener el grupo en "orden” y, además, se evidencia la aplicación del "espejo de clase" que consiste en asignar un lugar fijo para cada estudiante durante todo el año o un período que considere pertinente la persona docente: uso y abuso que provoca la monotonía y la consecuente desmotivación.

Se observó que la mayoría del cuerpo docente desarrolla todo su trabajo desde el escritorio, con pocos movimientos que se circunscriben a escribir algo en la pizarra, y son muy pocas las ocasiones en que se mueven por el aula y se acercan a los puestos individuales de sus estudiantes, lo que evidenciaría, con esta última forma de actuar, una mayor concentración y atención del estudiantado y más consultas pertinentes. 


\section{Reflexiones sobre el tema}

Se hace necesario referirse a un perfil de desempeño docente en relación con la ecología de los espacios vitales de aprendizaje, teniendo en cuenta que el cuerpo docente de sétimo año pueda desarrollar el perfil adecuado (véase la Tabla 1).

Tabla 1

Perfil del cuerpo docente según la dimensión ecológica de los espacios educativos

\begin{tabular}{|c|c|}
\hline $\begin{array}{l}\text { Sobre la organización y la } \\
\text { distribución del grupo en el } \\
\text { espacio físico }\end{array}$ & $\begin{array}{l}\text { - Diagnosticar la etapa del desarrollo maduracional y el comportamiento } \\
\text { en que la comunidad estudiantil adolescente se encuentra, y considerar la } \\
\text { diversidad de características que presenta el grupo, para, así, adecuar su } \\
\text { labor como docente a las necesidades que esa presenta. } \\
\text { - Tomar en cuenta que el estudiantado responde en clase de distinta manera, } \\
\text { de acuerdo con la motivación que se le dé, sin olvidar que dentro de las } \\
\text { prioridades en este grupo de edad no se encuentra lo académico. } \\
\text { - Permitir a la comunidad estudiantil el libre agrupamiento por afinidad e } \\
\text { intereses, y que se pueda propiciar reagrupamientos acordes con el desa- } \\
\text { rrollo armónico y constructivo del colectivo. } \\
\text { - Seleccionar estrategias para ordenar el grupo de acuerdo con el propósito } \\
\text { de la clase, captar la atención del estudiantado y evitar la dispersión; y } \\
\text { saber diferenciar entre los efectos de estrategias permisivas, amenazadoras } \\
\text { y, por el contrario, firmes y gratificantes. }\end{array}$ \\
\hline $\begin{array}{l}\text { Sobre la disposición de los } \\
\text { recursos materiales }\end{array}$ & $\begin{array}{l}\text { - Reinventar nuevas formas para desarrollar los contenidos de la clase, de } \\
\text { acuerdo con la dinámica que cada grupo tiene, según su tamaño y su diver- } \\
\text { sidad, y considerar los recursos de la institución, el tiempo disponible y la } \\
\text { respuesta que se espera del estudiantado. } \\
\text { - Maximizar el uso de nuevas tecnologías para la comunicación, disponibles } \\
\text { en los centros educativos y accesibles para la población docente y estudian- } \\
\text { til. }\end{array}$ \\
\hline $\begin{array}{l}\text { Sobre el manejo del tiempo } \\
\text { para el aprendizaje }\end{array}$ & $\begin{array}{l}\text { - Enlazar conocimientos previos con los nuevos, ya que la atención y la } \\
\text { comprensión de un tema nuevo por parte del estudiantado se favorece } \\
\text { si se dominan temáticas precedentes; es importante que quien ejerce la } \\
\text { docencia procure completar un tema de estudio, antes de iniciar temas } \\
\text { nuevos. } \\
\text { - Orientar el trabajo de la comunidad estudiantil, mediante la claridad } \\
\text { en la secuencia y el itinerario de lo que se impartirá en la clase, de } \\
\text { manera que se aproveche el tiempo de atención para el aprendizaje y } \\
\text { el cumplimiento de las tareas por realizar; mientras que los momentos } \\
\text { de distracción podrían ser para el descanso o los cambios de tema o } \\
\text { actividad. }\end{array}$ \\
\hline
\end{tabular}

Continúa $\ldots$ 


\begin{tabular}{|c|c|}
\hline $\begin{array}{l}\text { Sobre el ambiente y el clima } \\
\text { de convivencia }\end{array}$ & $\begin{array}{l}\text { - Ejercitar y consolidar la paciencia y la tolerancia como características } \\
\text { indispensables para: direccionar, constantemente, el comportamiento del } \\
\text { estudiantado en clase, mantener el orden, recordar las tareas por cumplir } \\
\text { y aportar a la clase, repetir instrucciones y procedimientos para resolver } \\
\text { ejercicios, ayudar a organizarse en el cumplimiento de las asignaciones que } \\
\text { las distintas materias requieren y favorecer su transición de la escuela al } \\
\text { colegio, recordar el cumplimiento del uso adecuado del uniforme y la pre- } \\
\text { sentación personal, así como el acatamiento de la normativa institucional. } \\
\text { - Propiciar un clima de apertura y confianza en el contacto entre docente y } \\
\text { población estudiantil, en el que se pueda dar y recibir, y expresar las nece- } \\
\text { sidades de forma natural, respetando, mutuamente, las ideas, con apertura } \\
\text { al diálogo asertivo y con escucha activa, sin juzgar y sin sentirse juzgado. } \\
\text { - Posibilitar el intercambio de ideas con una concepción de disciplina en } \\
\text { un ambiente integrador, que desarrolle el potencial humano y en el que la } \\
\text { persona docente ofrezca pautas para organizar el grupo (esta concepción se } \\
\text { aleja de la de "grupos callados y concentrados en sus tareas" u organizados } \\
\text { en estándares). } \\
\text { - Promover la autodisciplina y la autoorganización como habilidades para la } \\
\text { vida que favorecen la autonomía del grupo y el sentido de pertenencia. } \\
\text { - Implementar una mediación pedagógica atractiva a la población estudiantil, } \\
\text { teniendo presente que la persona docente no, necesariamente, tiene el con- } \\
\text { trol de todo lo que pasa en el aula. } \\
\text { - Detectar los liderazgos en el colectivo de estudiantes y mediar para su } \\
\text { orientación positiva, de manera que contribuya a la construcción cognitiva, } \\
\text { la cohesión y el buen ambiente grupal, aceptar nuevas y diferentes interac- } \\
\text { ciones. } \\
\text { - Colaborar en las actividades académicas, culturales, deportivas, recreativas } \\
\text { ción estudiantil que trascienda el espacio físico y el contexto institucional. } \\
\text { cambios inesperados o indeseables, tanto en el desarrollo de las lecciones, } \\
\text { como en los planes y los programas de estudio. }\end{array}$ \\
\hline $\begin{array}{l}\text { Sobre la atención a la } \\
\text { diversidad }\end{array}$ & $\begin{array}{l}\text { - Distinguir modelos de distribución y organización del aula, de acuerdo con } \\
\text { la ecología de los espacios de aprendizaje y las interacciones personales } \\
\text { que se quieran propiciar, y la integración de estudiantes que no logren rea- } \\
\text { lizarlo por su motivación o por sus necesidades específicas. } \\
\text { - Promover el contacto con la población estudiantil que favorezca un ambien- } \\
\text { te de aula integrador, de trabajo colaborativo y cooperativo, en el que ellos } \\
\text { y ellas tengan sentido de pertenencia. }\end{array}$ \\
\hline
\end{tabular}

Nota. Proyecto Perfiles dinámicas y desafíos de la educación costarricense (2008) Informe de investigación Etapa III. UNA-CIDE. 
Se puede concluir que el desempeño docente, implicado en este perfil, contribuye al desarrollo de potencialidades, al fortalecimiento de las relaciones interpersonales, a la cooperación y a la construcción colectiva de aprendizajes, en una ecología del espacio vital realmente motivadora y acogedora.

\section{Referencias bibliográficas}

Alfaro, M., Brenes, A., Gamboa, A., Jiménez, S., Martín, J., Ramírez, A., et al. (2008). Construcción del perfil de desempeño docente para el sétimo año de la educación pública. Informe Final de Investigación. Heredia: Universidad Nacional.

Bisquerra, R. (coord.) (1998). Modelos de orientación e intervención psicopedadógica. Barcelona, España: Praxis.

Boeglin, M. (2008). Leer y redactar en la universidad. Del caos de las ideas al texto estructurado: Bogotá, Colombia: Eduforma.

Bronfenbrenner, U. (1987). La ecología del desarrollo humano. Barcelona, España: Paidós.

Candegabe, S. (2001). Ideas para organizar el aula. Recuperado el 30 de abril, 2007, de www. turemanso.com.ar/fuego/educacion/organizaraula.html

García, N., Rojas, M., Campos, N., Brenes, M. \& Campos, E. (1993). Conocimiento, participación y cambio: capacitación a docentes a partir de la investigación en el aula. San José, Costa Rica: EUCR.

Morin, E. (1999). Los siete saberes necesarios para la educación del futuro. París, Francia: UNESCO.

\section{Otras fuentes consultadas}

Aparicio, E. (2007). Metodologías cambiantes en espacios de mejora de la calidad universitaria. Recuperado el 27 de febrero, 2008, de http://www.eduonline.ua.es/jornadas2007/ comunicaciones/2C4.pdf?PHPSESSID $=$ cbccbdf7bdf77780f5153163c2f16c2a

Bascuñan, J. \& López, M. (1998). Observación de procesos educativos en el aula. Psicología ecología aplicada a la sala de clase. Recuperado el 27 de febrero, 2008, de www.sectormatematica. cl/pedagogía/07\%20OBservacion\%20DE\%20PROCESOS\%20EDUCATIVOS\%20EN\%20 EL\%20AULA.DOC

Cortés, A. (2002). La contribución de la psicología ecológica al desarrollo moral. Un estudio con adolescentes. Recuperado el 27 de febrero, 2008, de www.um.es/analesps/v18/v18 1/0718 1.pdf 
García, F. A. (2001). Mesa Redonda: Conceptualización del desarrollo y la atención temprana desde las distintas escuelas psicológicas. Modelo ecológico/modelo integral de intervención en atención temprana. Recuperado el 27 febrero, 2008, de www.paidos.rediris.es/genysi/ actividades/jornadas/xijorp/xi_Garcia.pdf

Gómez, L. F. (2003). Las teorías implícitas de los profesores y sus acciones en el aula. Recuperado el 24 de enero, 2008, de www.eva.iteso.mx/trabajos/lgomez/ponenciateoriasimplicitas.pdf

Oliver, J. (1989). Espacios educativos y sistemas de formación. Metodología ecológica y organización educativa. Recuperado el 24 de enero, 2008, de www.dialnet.unirioja.es/servlet/fichero articu $\underline{\text { lo? codigo }=117613 \& \text { orden }=61997}$

Ribaya, F. (2007). La ausencia a clase. Recuperado el 24 de enero, 2008, de www.quadernsdigitals. net/index.php?accionMenu=hemeroteca.VisualizaArticuloIU.visualiza\&articulo id=10209 